\title{
Providers adherence to essential contents of antenatal care services increases birth weight in Bahir Dar City Administration, north West Ethiopia: a prospective follow up study
}

Tadese Ejigu Tafere ${ }^{1,2^{*}}$, Mesganaw Fanthahun Afework ${ }^{1}$ and Alemayehu Woreku Yalew ${ }^{1}$

\begin{abstract}
Background: Low birth weight (LBW) is one of the most important factors affecting child morbidity and mortality worldwide. Antenatal care (ANC) is an opportunity for reaching pregnant women with a number of interventions that may be vital to their health and well-being of their infants. However, data on the link between ANC quality and LBW remain limited especially in developing countries. Therefore, this study was aimed at investigating the effect of ANC service quality on birth weight among pregnant women attending ANC at public health facilities of Bahir Dar City Administration, Bahir Dar, Ethiopia using provision of essential services by providers as proxy for quality of care.
\end{abstract}

Methods: Nine hundred seventy pregnant women with gestational age $\leq 16$ weeks who came for their first ANC visit and selected by systematic sampling were enrolled and followed until delivery. Longitudinal data was collected during consultation with ANC providers using structured observation checklist. Women who gave birth at home and those who deliver a premature or still birth baby were excluded as data on birth weight could not be obtained for home deliveries and as the birth weight of the baby might be affected due to prematurity and still birth. Completed data were obtained from 718 women (since the rest women gave birth at home, we could not obtain birth weight data and we exclude them from analysis). The overall ANC service was considered as acceptable quality if women received $\geq 75$ th percentile of the essential ANC services. Generalized Estimating Equation was carried out to identify predictors of birth weight by controlling the cluster effect among women who received ANC services in the same facility.

Results: The prevalence of low birth weight $(<2500 \mathrm{~g})$ was $7.8 \%(95 \% \mathrm{Cl}=6.0 \%, 9.7 \%)$ with $1.4 \%$ versus $10.5 \%$ among those who received acceptable and not acceptable quality ANC services respectively, $P$-value $<0.001$. Maternal nutritional advice, iron-folic acid supplementation, tetanus toxoid vaccination, maternal educational status, parity and age were determinants for birth weight.

Conclusion and recommendation: The study showed that access to quality ANC services led to good birth weight outcome. Strengthening adherence of providers to essential components of antenatal care through regular monitoring and need based capacity building is very important for reducing the risk of low birth weight.

Keywords: Quality of antenatal care, Birth weight, Pregnancy outcome, Neonatal mortality

\footnotetext{
* Correspondence: tade_et@yahoo.com

${ }^{1}$ School of Public Health (SPH), College of Health Sciences, Addis Ababa University, Addis Ababa, Ethiopia

${ }^{2}$ School of Public Health, College of Medicine and Health Sciences, Bahir Dar University, Bahir Dar, Ethiopia
}

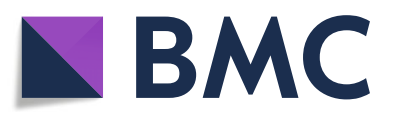

(c) The Author(s). 2018 Open Access This article is distributed under the terms of the Creative Commons Attribution 4.0 International License (http://creativecommons.org/licenses/by/4.0/), which permits unrestricted use, distribution, and reproduction in any medium, provided you give appropriate credit to the original author(s) and the source, provide a link to the Creative Commons license, and indicate if changes were made. The Creative Commons Public Domain Dedication waiver (http://creativecommons.org/publicdomain/zero/1.0/) applies to the data made available in this article, unless otherwise stated. 


\section{Plain English summary}

A child's birth weight or size at birth is an important indicator of the child's vulnerability to the risk of childhood illnesses and the child's chances of survival. Low birth weight (LBW) contributes to $60 \%$ to $80 \%$ of all neonatal deaths. Though this is the fact, based on the 2016 Ethiopian demographic and Health survey report, the prevalence of LBW in Ethiopia is $13.4 \%$. It is also worth noting the prevalence might be higher as only $14 \%$ of children in Ethiopia are weighed at birth. However, data on the link between ANC quality and LBW remain limited especially in low income countries like Ethiopia.

Pregnant women with gestational age $\leq 16$ weeks who came for their first ANC visit and selected by systematic random sampling were enrolled and followed until delivery. Longitudinal data were collected during consultation with ANC providers to assess the quality of ANC services during their four focused ANC visits using structured observation checklist. The overall ANC service was considered as acceptable quality if women received $\geq 75$ th percentile of the essential ANC services.

Among 970 enrolled pregnant women, completed data for this study was obtained from 718 women (as the rest women give birth at home they are excluded from the analysis due to lack of data on birth weight).

The prevalence of low birth weight $(<2500 \mathrm{~g})$ for the current study was $7.8 \%(95 \% \mathrm{CI}=6.0 \%, 9.7 \%)$ with $1.4 \%$ versus $10.5 \%$ among those who received acceptable and not acceptable quality ANC services respectively. This indicates quality ANC service has a better birth weight outcome, that implied a woman's contact with her ANC provider should be more than a simple visit.

\section{Background}

The World Health Organization (WHO) has defined low birth weight (LBW) as the weight of live born infants of $<2500$ g regardless of gestational age or any other factor that might have an influence on it. A child's birth weight or size at birth is an important indicator of the child's vulnerability to the risk of childhood illnesses and the child's chances of survival. The global prevalence of LBW is in the range between 15 and 20\%, which accounts to about 20 million infants born; approximately one third of neonatal deaths are attributable to LBW [1].

The prevalence of LBW in Ethiopia and Amhara region in particular was 11 and $11.2 \%$ respectively. It is also worth noting these rates might be higher as only $5 \%$ of children in Ethiopia are weighed at birth [2]. The goal set by WHO is to achieve a $30 \%$ reduction of the number of infants born with a weight $<2500 \mathrm{~g}$ by the year 2025; 3.9\% relative reduction per year between 2012 and 2025 [3].
Antenatal care (ANC) is an opportunity for reaching pregnant women with a number of interventions that may be vital to their health and well-being of their infants [4]. Its putative benefits to babies include increased intra uterine growth, reduced risk of infection and increased survival [5].

The assessment of prenatal care uptake and quality are essential steps toward improving accessibility and birth outcomes. Some elements of the ANC package (Iron folic acid supplementation, nutritional advice, tetanus toxoid vaccination, screening for pre-eclampsia, screening and treatment of asymptomatic bacteriuria and syphilis) have been shown to be cost-effective in a Sub-Saharan African context [6, 7].

Even though the prevalence of LBW is higher in low and middle income countries, most of the research on the link between antenatal care and birth weight has been conducted in high income nations [1] and many of these were cross sectional studies that focus on assessing the association between frequency (uptake) of antenatal visits rather than adherence of providers to essential services of antenatal care and birth weight. In addition, low income countries usually follow guidelines based on research findings conducted in high income nations. However, the results from research conducted in these countries are not necessarily applicable to low income nations. Therefore, the aim of this study was to investigate the association between adherences of providers to essential services of antenatal care and birth weight which might have an input for the design of guidelines and policies to improve child survival in low income countries like Ethiopia.

\section{Methods}

\section{Study design, setting and population}

A facility based prospective follow up study was conducted from October 2015 to August 2016 in Bahir Dar City Administration, Amhara National Regional State, which is located in the North West part of Ethiopia. According to the Amhara National Regional State Bureau of Finance and Economic Development report, the projected population by $2015 / 16$ was 297,775 (80.5\% urban Vs $19.5 \%$ rural), of these 156,515 (52.6\%) were females and there were 10,035 eligible pregnant women in the same year [8].

The study was conducted on seven public health facilities, one hospital and six health centers. All selected first visit pregnant women with gestational age $\leq$ 16weekss and attending ANC service during data collection period that were voluntary to participate verbal informed consent were included in the study. Women who gave birth at home were excluded as data on birth weight could not be obtained. In addition, those who gave a premature or still-birth baby; those who had any 
medical complication while pregnant (diabetes mellitus, heart disease, eclamptic and multiple pregnancy or give birth by caesarian section) were excluded as the birth weight of the baby might be affected due to the underlying conditions.

\section{Sample size and sampling technique}

The current study is part of a large follow up study with multiple objectives. The detail of the sample size calculation assumptions to address all objectives is described in the other part of the study published on Plos ONE [9]. For the current study a sample size was calculated with the predicted incidence of LBW babies as 6\% among mothers completing four antenatal visits (compared to $25 \%$ among mothers with less than four visits) [10], and with $80 \%$ power, $95 \%$ confidence level and $15 \%$ non-response rate. The final calculated sample size was 304. However, to increase the power of the study, all 718 women who deliver a singleton baby at the study health facilities were included in the analysis.

\section{Data collection procedure}

A structured observation check list to measure ANC quality was developed based on focused antenatal care protocol and used to observe the routine ANC practice for each visit. The weight of naked neonates was measured in kilograms to the nearest $100 \mathrm{~g}$ by using standard beam balance in the delivery room immediately after birth (preferably within 1 hour) by trained diploma midwives. Seven female diploma Midwives and two Bachelor of Science female Midwives were recruited as data collectors and supervisors respectively. Both data collectors and supervisors were not working in health facilities under the study. Training was given on the data collection instrument and how to approach and observe the ANC service provision. Pretest of the instrument, close supervision and daily checkup of the filled questionnaire for completeness were also done to maintain the data quality.

\section{Dependent variable}

Birth weight in kilograms; which was measured within 1 hour after birth.

\section{Independent variable}

Quality of ANC service was the main exposure variable.

Quality of ANC service was measured based on the interventions that the mother received during antenatal care: laboratory tests done like blood and urine tests, physical examinations like weight, height, blood pressure, providing TT vaccination, iron - folic acid supplementation, deworming, nutritional advice, information on immunization and breast feeding.
If the essential ANC service was given it was coded as 1 otherwise 0 and a composite index for the overall ANC service quality was calculated. Percentile was computed to categorize the quality of the services a woman received. If she scored $\geq 75$ th percentile, of essential ANC services, ANC service was considered acceptable quality otherwise not [11].

\section{Data analysis}

Data were coded and entered into EPI data version3.1 and exported to SPSS version 20 for analysis. Descriptive statistics were used to describe the data. Generalized Estimating Equation linear regression analysis with robust estimator and exchangeable working correlation matrix was carried out to control the cluster effect of women who received ANC services within the same facility and to identify the predictor variables for birth weight of neonates. A $p$-value $<0.2$ was considered to retain variables for the multivariable Generalized Estimating Equation linear regression analysis and $P$-value $<0.05$ was considered to identify statistically significant predictors for birth weight.

\section{Results}

\section{Characteristics of ANC attendants}

Among 718 women who gave birth at health facilities, those with age $\leq 19$ years (teen age pregnancy) and those with age $\geq 35$ years (elderly pregnancy) were 4.9 and $4.0 \%$ respectively. The mean ( \pm standard deviation) age at birth was $25.32 \pm 4.21$ years. Above $90 \%$, (94.2\%) of women were urban residents and more than half of them $(52.9 \%)$ were house wives. Above $90 \%$, (90.9\%) of study participants were Orthodox Christian and almost all women (98.8\%) were currently married. With regard to their educational status, $11.4 \%$ of women had no formal education while $63.3 \%$ of women had joined secondary school and above (Table 1).

Greater than two out of five of the pregnant women, 326 (45.4\%) were primi-para; while more than one out of two $378(52.6 \%)$ of women were multi-para and 14 $(1.9 \%)$ of them were grand multi para.

\section{Adherence to essential contents of antenatal care and birth weight}

Above $95 \%$, 701(97.6\%) of pregnant women received two doses of tetanus toxoid vaccine but the rest 17 (2.4\%) missed one dose. About two third (57.1\%) of the pregnant women were counselled about BPCRP at least once in their four ANC visits. The information provision on maternal nutrition, breast feeding and immunization was also heterogeneous (Table 2).

With regard to iron supplementation, 304 (42.3\%), 327 (45.5\%), $77(10.7 \%)$ and $10(1.4 \%)$ of pregnant women were supplemented with 120,90, 60 and 30 tablets of 
Table 1 Socio-demographic characteristics and birth weight among study participants in public health facilities of Bahir Dar City administration ( $N=718$ ), October 2015 to August 2016

\begin{tabular}{|c|c|c|c|c|}
\hline \multirow[b]{2}{*}{ Variables } & \multicolumn{3}{|l|}{ Birth weight } & \multirow{2}{*}{$\begin{array}{l}\text { Total } \\
\text { frequency }\end{array}$} \\
\hline & low & normal & over weight & \\
\hline \multicolumn{5}{|l|}{$\overline{\text { Age }}$} \\
\hline$\leq 19$ & $0(0.0 \%)$ & $34(97.1 \%)$ & $1(2.9 \%)$ & $35(100.0 \%)$ \\
\hline $20-24$ & $20(7.0 \%)$ & $260(91.5 \%)$ & $4(1.4 \%)$ & $284(100.0 \%)$ \\
\hline $25-29$ & $16(5.9 \%)$ & $245(9.1 \%)$ & $8(3.0 \%)$ & $269(100.0 \%)$ \\
\hline $30-34$ & $7(7.0 \%)$ & $93(92.1 \%)$ & $1(0.9 \%)$ & $101(100.0 \%)$ \\
\hline$\geq 35$ & $13(44.8 \%)$ & $16(55.2 \%)$ & $0(0.0 \%)$ & $29(100.0 \%)$ \\
\hline \multicolumn{5}{|l|}{ Residence } \\
\hline Urban & $43(6.4 \%)$ & $619(91.6 \%)$ & $14(2.1 \%)$ & $676(100.0 \%)$ \\
\hline Rural & $13(31.0 \%)$ & $29(69.0 \%)$ & $0(0.0 \%)$ & $42(100.0 \%)$ \\
\hline \multicolumn{5}{|l|}{ Educational status } \\
\hline No formal education & $23(20.9 \%)$ & $86(78.2 \%)$ & $1(0.9 \%)$ & $110(100.0 \%)$ \\
\hline Primary & $15(9.8 \%)$ & $138(90.2 \%)$ & $0(0.0 \%)$ & $153(100.0 \%)$ \\
\hline Secondary & $18(4.0 \%)$ & $424(93.1 \%)$ & $13(2.9 \%)$ & $455(100.0 \%)$ \\
\hline \multicolumn{5}{|l|}{ Occupation } \\
\hline Farmer & $13(32.5 \%)$ & $27(67.5 \%)$ & $0(0.0 \%)$ & $40(100.0 \%)$ \\
\hline House wife & $31(8.2 \%)$ & $346(91.0 \%)$ & $3(0.8 \%)$ & $380(100.0 \%)$ \\
\hline Employee $^{a}$ & $12(4.0 \%)$ & $275(92.3 \%)$ & $11(3.7 \%)$ & $298(100.0 \%)$ \\
\hline \multicolumn{5}{|l|}{ Religion } \\
\hline Orthodox & $51(7.8 \%)$ & $589(90.2 \%)$ & $13(2.0 \%)$ & $653(100.0 \%)$ \\
\hline Muslim & $5(7.9 \%)$ & $57(90.5 \%)$ & $1(1.6 \%)$ & $63(100.0 \%)$ \\
\hline Protestant & $0(0.0 \%)$ & $2(100.0 \%)$ & $0(0.0 \%)$ & $2(100.0 \%)$ \\
\hline \multicolumn{5}{|l|}{ Marital status } \\
\hline Married & $54(7.6 \%)$ & $641(90.4 \%)$ & $14(2.0 \%)$ & 709 (100.0\%) \\
\hline Divorced & 1 (12.5\%) & 7 (87.5\%) & $0(0.0 \%)$ & $8(100.0 \%)$ \\
\hline Widowed & 1 (100.0\%) & $0(0.0 \%)$ & $0(0.0 \%)$ & 1 (100.0\%) \\
\hline \multicolumn{5}{|l|}{ Ethnicity } \\
\hline Amhara & 55 (8.1\%) & $619(90.2 \%)$ & $12(1.7 \%)$ & $686(100.0 \%)$ \\
\hline Tigre & 1 (4.5\%) & 19 (86.4\%) & $2(9.1 \%)$ & $22(100.0 \%)$ \\
\hline Awi & $0(0.0 \%)$ & 7 (100.0\%) & $0(0.0 \%)$ & 7 (100.0\%) \\
\hline Oromo & $0(0.0 \%)$ & $3(100.0 \%)$ & $0(0.0 \%)$ & $3(100.0 \%)$ \\
\hline Grand total & $56(7.8 \%)$ & 648 (90.3\%) & 14 (1.9\%) & 718 (100.0\%) \\
\hline
\end{tabular}

a governmental employees, nongovernmental employees, daily laborers and waiters

Table 2 Information provided to pregnant women during ANC visits at public health facilities of Bahir Dar City Administration, Bahir Dar, Ethiopia; October 2015 to August 2016 ( $N=718)$

\begin{tabular}{lllll}
\hline ANC services given & During 1st visit, $\mathrm{n}(\%)$ & During 2nd visit, $\mathrm{n}(\%)$ & During 3rd visit, $\mathrm{n}(\%)$ & During 4th visit, $\mathrm{n}(\%)$ \\
\hline Advised on maternal nutrition & $496(69.1)$ & $608(84.7)$ & $506(70.5)$ & $296(41.2)$ \\
Advised on breast feeding & - & - & $14(1.9)$ & $205(28.6)$ \\
Advised immunization & - & - & $11(1.5)$ & $196(27.3)$ \\
Checked conjunctiva for pallor & $575(80.1)$ & $238(33.1)$ & $507(70.6)$ & $272(37.9)$ \\
\hline
\end{tabular}


iron- folic acid respectively. In addition, 509 (70.9\%) and $564(78.6 \%)$ of pregnant women received anti-heliments for deworming and were advised to use insecticide treated bed net for prevention of malaria respectively (Table 3).

The mean ( \pm SD) birth weight of neonates was 3113.0 $( \pm 474.9)$ gram with the minimum and maximum birth weights of 1800 and $4400 \mathrm{~g}$ respectively. The mean birth weight of neonates among women who received acceptable and unacceptable quality of ANC service was 3232 $( \pm 0.478)$ and $3062( \pm 0.476)$ grams. Of the total pregnant women who deliver at health facilities, 56 (7.8\%; $95 \% \mathrm{CI}=6.0 \%, 9.7 \%)$ gave a low birth weight baby, 648 (90.3\%; $95 \% \mathrm{CI}=88.0 \%, 92.2 \%)$ gave a baby with normal birth weight $(2500-4000 \mathrm{~g})$ and $14(1.9 \% ; 95 \% \mathrm{CI}=1.0 \%$, $3.1 \%$ ) gave birth overweight babies (>4000 g).

\section{Association between contents of ANC service with birth weight}

In a bivariate Generalized Estimating Equation linear regression analysis, there was a positive association between birth weight of neonates with the maternal nutritional advice during ANC, Iron -folic acid supplementation, deworming with anti-heliments, doses of tetanus toxoid vaccination of the mother, Venereal Disease Research Laboratory (VDRL) test of the mother, hematocrit test of the mother at first ANC visit, hematocrit test of the mother at fourth ANC visit, advice on Insecticide Treated Bed Net (ITN) use and most of the socio-demographic factors such as residence, maternal age, educational status, occupation and parity. Therefore, all these variables were fitted to the multivariable GEE linear regression analysis.

In multivariable Generalized Estimating Equation linear regression analysis, maternal nutritional advice during ANC, Iron supplementation, tetanus toxoid vaccination, educational status and parity remained to have statistically significant association with birth weight. In addition, deworming with anti-heliments, advice on ITN use, having VDRL test, having hemoglobin test at fourth ANC visits and living in urban areas showed an increase in birth weight but the association was not statistically significant.

When the frequency of maternal nutritional advice during ANC visits was increased by one, the birth weight of a neonate was increased by $147 \mathrm{~g}(\beta=0.147,95 \% \mathrm{CI}=$ $0.11,0.19)$. When the number of iron tablet supplementation to pregnant women during ANC visits decreased from 90 or more tablets to less than 90 tablets, the birth weight of their neonates decreased by $358 \mathrm{~g}(\beta=-0.358$, $95 \% \mathrm{CI}=-0.476,-0.240)$. When the dose of tetanus toxoid vaccine given to pregnant women during ANC visits increased from one to two doses till delivery, the birth weight of their neonates increased by $609 \mathrm{~g}(\beta=0.609$, $95 \% \mathrm{CI}=0.316,0.903)$. When the educational status of women increased by one unit, the birth weight also increased by $79 \mathrm{~g}(\beta=0.079,95 \% \mathrm{CI}=0.06,0.10)$. When the parity of women increased by one the birth weight of their neonates decreased by $174 \mathrm{~g}(\beta=-0.174,95 \% \mathrm{CI}$ $=0.24,0.11)$ (Table 4).

Table 3 Birth weight status of neonates among pregnant women who received different ANC services at public health facilities of Bahir Dar City Administration, Bahir Dar, Ethiopia; October 2015 to August 2016 ( $N=718)$

\begin{tabular}{|c|c|c|c|c|c|}
\hline Variables & & $\begin{array}{l}\text { Low birth weight, } \\
\mathrm{n}(\%)\end{array}$ & $\begin{array}{l}\text { Normal birth weight, } \\
\mathrm{n}(\%)\end{array}$ & $\begin{array}{l}\text { Overweight, } \\
\mathrm{n}(\%)\end{array}$ & $\begin{array}{l}\text { Total, } \\
\text { n (\%) }\end{array}$ \\
\hline \multirow[t]{2}{*}{ Quality of ANC } & Received acceptable quality & $3(1.4 \%)$ & $203(94.4 \%)$ & $9(4.2 \%)$ & $215(100.0 \%)$ \\
\hline & Received not acceptable quality & $53(10.5 \%)$ & $445(88.5 \%)$ & $5(1.0 \%)$ & $503(100.0 \%)$ \\
\hline \multirow[t]{5}{*}{ Maternal nutritional advice } & Not advised at all & $4(13.3 \%)$ & $25(83.3 \%)$ & $1(3.3 \%)$ & $30(100.0 \%)$ \\
\hline & Advised in one visit & $7(53.8 \%)$ & $6(46.1 \%)$ & $0(0.0 \%)$ & $13(100.0 \%)$ \\
\hline & Advised in two visits & $30(11.7 \%)$ & $225(87.5 \%)$ & $2(0.8 \%)$ & $257(100.0 \%)$ \\
\hline & Advised in three visits & $12(4.1 \%)$ & 275 (93.8\%) & $6(2.0 \%)$ & $293(100.0 \%)$ \\
\hline & Advised in four visits & $3(2.4 \%)$ & 117 (93.6\%) & $5(4.0 \%)$ & $125(100.0 \%)$ \\
\hline \multirow{4}{*}{$\begin{array}{l}\text { Iron - folic acid } \\
\text { supplementation }\end{array}$} & Given 30 tablets & $9(90.0 \%)$ & $1(10.0 \%)$ & $0(0.0 \%)$ & $10(100.0 \%)$ \\
\hline & Given 60 tablets & $18(23.4 \%)$ & $59(76.6 \%)$ & $0(0.0 \%)$ & $77(100.0 \%)$ \\
\hline & Given 90 tablets & $15(4.7 \%)$ & $307(93.8 \%)$ & $5(1.5 \%)$ & $327(100.0 \%)$ \\
\hline & Given 120 tablets & $14(4.5 \%)$ & $281(92.5 \%)$ & $9(3.0 \%)$ & $304(100.0 \%)$ \\
\hline \multirow[t]{2}{*}{ Deworming } & Not given & $16(7.7 \%)$ & $191(91.3 \%)$ & $2(1.0 \%)$ & $209(100.0 \%)$ \\
\hline & Given at 3rd/4th visit & $40(7.9 \%)$ & $457(89.8 \%)$ & $12(2.4 \%)$ & $509(100.0 \%)$ \\
\hline \multirow[t]{2}{*}{ Tetanus toxoid vaccine } & Given one dose & $12(70.6 \%)$ & $5(29.4 \%)$ & $0(0.0 \%)$ & $17(100.0 \%)$ \\
\hline & Given two doses & $44(6.3 \%)$ & $643(91.7 \%)$ & $14(2.0 \%)$ & $701(100.0 \%)$ \\
\hline
\end{tabular}




\section{Discussion}

World Health organization set a goal to reduce the number of infants with LBW by 30\% by the year 2025 and similarly the Sustainable Development Goal (SDG) by 2030 also aimed to reduce neonatal mortality to at least as low as 12 per 1000 live births. Though LBW contributes to $60 \%$ to $80 \%$ of all neonatal deaths [3, 12]; In this study about $7.8 \%(95 \% \mathrm{CI}=6.0-9.7 \%$ pregnant women gave low birth weight baby. This finding is consistent with other study findings in Ethiopia, 9\%in urban population and $11 \%$ in the general population [2], in Ghana (6.1\%) [13], in Colombia (8.7\%) [14], in Mexico (8\%) [15] but is lower than study findings in Angola and (25.9\%) [16], in India (26.8\%) [17].

The possible reason might be due to the difference in the characteristics of study participants as all the participants in this study were enrolled if they started their ANC in their first trimester and were also followed till their four antenatal visits and most (94\%) were from urban and peri- urban populations that might increase the tendency to get the essential components of antenatal care like maternal nutritional advice, Iron-folic acid supplementation and other services during subsequent
ANC visits that might contribute positively for birth weight. The difference in the study period might also have its own effect for the difference in the findings as many interventions like the community based newborn care packages for improving maternal and newborn health are being implemented by different countries including Ethiopia. In addition, except the current study and the study in Ghana all other studies collect information about birth weight based on mother's recall or birth registration records that might affect accuracy of their result.

This study revealed that the frequency of nutritional advice given to pregnant women during their ANC visits have statistically significant effect on increasing the birth weight of their babies. In contrary to this finding a meta-analysis done from thirty-three studies evidenced nutrition education and counselling significantly improved mean birth weight but was not significantly associated with the risk of low birth weight [18]. The difference might be due to the reason that this study considers dose -response relationship of nutritional advice unlike those studies included in the meta- analysis and the authors of the meta- analysis also indicated

Table 4 Multivariable Generalized Estimating Equation linear regression to identify determinants of birth weight among pregnant women attending ANC at public health facilities of Bahir- Dar City Administration ( $n=718$ ), October 2015 to August 2016

\begin{tabular}{|c|c|c|c|c|c|}
\hline \multicolumn{2}{|l|}{ Dummy variables } & Number (\%) & $\begin{array}{l}\text { Un-Standardized } \beta \text { with } \\
95 \% \mathrm{Cl}\end{array}$ & $\begin{array}{l}\text { Standardized } \beta \text { with } \\
95 \% \mathrm{Cl}\end{array}$ & $P$-value \\
\hline \multicolumn{2}{|c|}{ Maternal nutritional advice during ANC } & $718(100)$ & $0.147(0.11,0.19)$ & $0.138(0.10,0.17)$ & $0.001^{*}$ \\
\hline \multirow[t]{2}{*}{ Residence } & Urban & $676(94.2)$ & $0.398(0.249,0.547)$ & $0.122(-0.02,0.26)$ & 0.09 \\
\hline & Rural & $42(5.8)$ & $0^{\mathrm{a}}$ & $0^{a}$ & \\
\hline \multirow[t]{2}{*}{ Iron supplementation } & Took $<9$ o tabs & $87(12.1)$ & $-0.358(-0.476,-0.240)$ & $-0.240(-0.34,-0.14)$ & $0.001^{*}$ \\
\hline & Took $\geq 90$ tabs & $631(87.9)$ & $0^{\mathrm{a}}$ & $0^{\mathrm{a}}$ & \\
\hline \multirow[t]{2}{*}{ TT vaccination } & Took two doses & $701(97.6)$ & $0.609(0.316,0.903)$ & $0.286(0.03,0.54)$ & $0.03^{*}$ \\
\hline & Took one dose & $17(2.4)$ & $0^{\mathrm{a}}$ & $0^{\mathrm{a}}$ & \\
\hline \multirow[t]{2}{*}{ Deworming at 3rd/4th visit } & Yes & $509(70.9)$ & $0.109(0.035,0.183)$ & $0.031(-0.04,0.11)$ & 0.41 \\
\hline & No & $209(29.1)$ & $0^{a}$ & $0^{\mathrm{a}}$ & \\
\hline \multirow[t]{2}{*}{ Hematocrit test at 1st visit } & Yes & $684(95.3)$ & $0.115(-0.041,0.271)$ & $-0.002(-0.15,0.15)$ & 0.98 \\
\hline & No & $34(4.7)$ & $0^{a}$ & $0^{a}$ & \\
\hline \multirow[t]{2}{*}{ Hematocrit test 3rd visit } & Yes & $378(52.6)$ & $0.121(0.052,0.190)$ & $0.026(-0.04,0.09)$ & 0.46 \\
\hline & No & $340(47.4)$ & $0^{\mathrm{a}}$ & $0^{\mathrm{a}}$ & \\
\hline \multirow[t]{2}{*}{ VDRL test } & Yes & $644(89.7)$ & $0.120(0.008,0.231)$ & $0.028(-0.08,0.14)$ & 0.61 \\
\hline & No & $74(10.3)$ & $0^{\mathrm{a}}$ & $0^{a}$ & \\
\hline \multirow[t]{2}{*}{ Advice about ITN use } & Yes & $564(78.6)$ & $0.069(-0.011,0.149)$ & $0.031(-0.05,0.11)$ & 0.42 \\
\hline & No & $154(21.4)$ & $0^{\mathrm{a}}$ & $0^{a}$ & \\
\hline \multicolumn{2}{|c|}{ Educational status of the mother } & $718(100 \%)$ & $0.079(0.06,0.10)$ & $0.064(0.05,0.08)$ & $0.001^{*}$ \\
\hline \multicolumn{2}{|l|}{ Maternal age } & $718(100 \%)$ & $-0.067(-0.13,-0.01)$ & $-0.037(-0.10,0.03)$ & 0.25 \\
\hline \multicolumn{2}{|l|}{ Parity } & $718(100 \%)$ & $-0.174(0.24,0.11)$ & $-0.092(-0.16,-0.03)$ & $0.01^{*}$ \\
\hline
\end{tabular}

*(indicates statistical significance at $p$-value $<0.05), 0^{\text {a }}$ indicates a reference category for the categorical predictor variables NB The data about birth weight were entered in kilograms to the data set and the betas are converted to grams by multiplying with 1000 for the ease of interpretation 
except one study, the overall quality of studies extracted were low. The other possible reason could be the difference since most of the studies included in the meta-analysis were conducted in developed countries (USA, UK, Australia, Argentina and Greece). The participants of studies in these countries might have a better educational status and awareness on maternal education during pregnancy and other essential services needed for a pregnant woman so that there might not be a significant difference between women who were counselled and those who were not counselled about maternal nutrition during ANC.

In low-income countries, $25 \%$ of low birth weight was attributable to maternal anemia during pregnancy [19]. In spite of this, the current study also showed more than one in ten pregnant women were supplemented with less than 90 tablets throughout their pregnancy period. Iron-folic acid supplementation had also a dose -response relationship with birth weight; this study found that the birth weight of babies among pregnant women who were not supplemented with Iron-folic acid tablets or those who were supplemented with $<90$ tablets during their ANC visits was significantly decreased compared to those who were supplemented with 90 tablets or more.

This finding is also supported with a Meta-analysis done from ten randomized control trials indicating $2 \%$ increase in birth weight for every two-fold increase in folate intake. Other studies conducted in Spain and Atlanta also revealed duration longer than 4 months of iron supplementation gave significant protective odds ratio for low birth weight and women with low folate intake $(\leq 240 \mathrm{mg} / \mathrm{d})$ had $>3$ times greater risk for low birth weight infant than women with folate intake $>240 \mu \mathrm{g} / \mathrm{d}$ respectively [20-23]. The physiological mechanism of iron supplementation on birth weight is not clearly understood, however, there are two hypotheses about improvements in birth weight due to iron supplements. First, iron deficiency anemia leads to changes in nor-epinephrine, cortisol and corticotrophin resulting in oxidative stress to fetal growth which is reduced by iron supplementation. Second, iron supplementation helps to improve appetite leading to improvement in the overall nutritional status of mother [24].

In this study majority (97.6\%) of pregnant women were given two doses of tetanus toxoid vaccinations compared to the Ethiopian Demographic and Health Survey report in 2016 revealed that only half of the pregnant women who gave birth 5 years prior to the survey took two doses (with $72 \%$ urban and $46 \%$ rural women) [25]. The current study also showed providing two doses of tetanus toxoid for pregnant women during their antenatal care visits significantly increased the birth weight of their babies compared to those women who were given only one dose. The increase in birth weight of babies born from women who received two doses of tetanus toxoid might not be due to the direct effect of tetanus toxoid vaccination on birth weight rather it might be due to the fact that when women came to health facility for TT vaccinations, they are more likely to get nutritional advices or iron-folic acid supplementations that contribute for better birth weight.

This study also found that there was an increase in birth weight as educational status of pregnant women increased from no education or primary education to secondary education and above. This is also supported by studies conducted in Bangladesh and Beirut [26, 27]. In contrary to maternal education that had positive association to birth weight, multi parity or grand multi parity of mothers and older age of the mother ( $\geq 35$ years) had negative association with birth weight.

Prospective follow up of pregnant women through observation while they get the ANC service; collecting information about birth weight of the neonate at the moment of birth and applying a generalized estimating equation analysis to control the cluster effect of women who received the ANC service in the same health facility could be taken as the strength of the study but excluding women who gave birth at home due to missing of birth weight data and Hawthorn effect could be the limitation as those women might have different characteristics.

\section{Conclusion and recommendations}

The study found that near to one in ten neonates were born with low birth weight. Access to quality antenatal care increases the birth weight of new born babies. Essential components of antenatal care (maternal nutritional advice, iron-folic acid supplementation, and tetanus toxoid vaccination) and socio- demographic variables like maternal educational status and parity were determinants for birth weight. Strengthening adherence of providers to essential components of antenatal care like nutritional advice, iron- folic acid supplementation and tetanus toxoid vaccination for every pregnant woman through regular monitoring and provision of feedback and need based capacity building is very important for reducing the risk of low birth weight.

\footnotetext{
Abbreviations

ANC: Antenatal Care; Cl: Confidence Interval; FANC: Focused Antenatal Care; GEE: Generalized Estimating Equation; ITN: Insecticide Treated Bed Net; LBW: Low Birth weight; SPSS: Statistical Packages for Social Sciences; TT: Tetanus Toxoid; VDRL: Venereal Disease Research Laboratory

\section{Acknowledgements}

The authors would like to thank Addis Ababa and Bahir Dar Universities for covering the transportation and Perdiem cost of data collectors and supervisors during the data collection process of the study. They also sincerely thank the study participants for their participation and the data collectors and supervisors for their unreserved effort to realize this study.
} 


\section{Funding}

Addis Ababa University and Bahir Dar University covered the transportation and Perdiem cost of data collectors and supervisors during the data collection process.

\section{Availability of data and materials}

The datasets used and/or analyzed during the current study are available from the corresponding author on reasonable request.

\section{Authors' contributions}

TE was involved in the conception, and design of the research project proposal; analysis, interpretation, report and manuscript writing. MF had been involved in the conception and design of the research project proposal and AW was involved in the conception, design of research project, analysis, interpretation and approval of manuscript writing. TE and AW read and approved the final manuscript

\section{Ethics approval and consent to participate}

The study protocol was approved by the Institutional Review Board (IRB) of the College of Health Sciences, Addis Ababa University meeting number 004/2015and an approval letter was written on the date of May 6,2015with protocol number 008/15/SPH. Letter of permission was obtained from Amhara Regional Health Bureau and Bahir-Dar City Administration Zonal Health Office. Both ANC clients and providers were informed about the purpose of the study and verbal informed consent was also obtained before data collection. Study participants had the right to refuse participation or terminate their involvement at any point during observation. The information collected is kept confidential. Furthermore, report writing does not refer a specific respondent with identifiers.

\section{Consent for publication}

This manuscript does not contain any individual person's data in the form of image or video. Hence consent for publication is not applicable.

\section{Competing interests}

The authors declare that they have no any financial or non-financial competing interests (political, personal, religious, ideological, academic, intellectual, commercial or any other).

\section{Publisher's Note}

Springer Nature remains neutral with regard to jurisdictional claims in published maps and institutional affiliations.

\section{Received: 15 May 2018 Accepted: 19 September 2018}

\section{Published online: 29 September 2018}

\section{References}

1. World Health Organization (WHO: Guidelines on optimal feeding of low birth weight infants in low-and middle-income countries. Geneva. 2011.

2. Centeral Statitical Agency of Ethiopia: Ethipoian Demographic and Health Survey (EDHS) Final Report 2011.

3. World Health Organization. Comprehensive implementation plan on maternal, infant and young child nutrition: sixty-fifth world health assembly Geneva. In: Resolutions and Decisions; 2012.

4. Abou-Zahr CL, Wardlaw TM: Antenatal care in developing countries: promises, achievements and missed opportunities: an analysis of trends, levels and differentials, 1990-2001. 2003.

5. Campbell OM, Graham W. The lancet maternal survival series steering group: strategies for reducing maternal mortality: getting on with what works. Lancet. 2006;368:1284-99.

6. Adam T, Lim S, Mehta S, Bhutta ZA, Fogstad H, Mathai M, Zupan J, Darmstadt GL. Achieving the millennium development goals for health cost effectiveness analysis of strategies for maternal and neonatal health in developing countries. Br Med J. 2005;331:1107-10.

7. Lambon-Quayefio MP, Owoo NS. Examining the influence of antenatal care visits and skilled delivery on neonatal deaths in Ghana. Appl Health Econ Health Policy. 2014;12(5):511-22.

8. Amhara National Regional State Bureau of Finance and Economic Development. Population projection report 2015.

9. Ejigu Tafere T, Afework MF, Yalew AW. Antenatal care service quality increases the odds of utilizing institutional delivery in Bahir Dar city administration, North Western Ethiopia: a prospective follow up study. PLoS One. 2018;13(2)

10. Ahmed Z, Khoja S, Tirmizi SS. Antenatal care and the occurrence of low birth weight delivery among women in remote mountainous region of Chitral, Pakistan. Pak J Med Sci. 2012;28(5):800-5.

11. Barber $\mathrm{S}$. Does the quality of prenatal care matter in promoting skilled institutional delivery? A study in rural Mexico. Matern Child Health J. 2006; 10(5):419-25.

12. Organization. WH: Strategies towards ending preventable maternal mortality (EPMM). Geneva. 2015.

13. Amoakoh-Coleman K-G, Agyepong A, Kayode GA, Grobbee DE, Ansah EK. Provider adherence to first antenatal care guidelines and risk of pregnancy complications in public sector facilities: a Ghanaian cohort study. BMC Pregnancy Childbirth. 2016;16:369.

14. Pinzón-Rondón AM, Gutiérrez-Pinzon V Madriñan-Navia H, Amin J, AguileraOtalvaro AP, Hoyos-Martínez A. Low birth weight and prenatal care in Colombia: a cross-sectional study. BMC Pregnancy Childbirth. 2015:15:118.

15. Servan-Mori E, Sosa-Rubí SG, Najera-Leon E, Darney BG. Timeliness, frequency and content of antenatal care: which is most important to reducing indigenous disparities in birth weight in Mexico? Health Policy Plan. 2016;31(4):444-53.

16. Nimi TFS, Costa D, Campos P, Barros H. Prenatal care and pregnancy outcomes: a cross-sectional study in Luanda, Angola. Int J Gynecol Obstet. 2016:135:572-8

17. Misra A, Ray S, Patrikar S. A longitudinal study to determine association of various maternal factors with neonatal birth weight at a tertiary care hospital. Med J Armed Force India. 2015;71:270-3.

18. Webb Girard A, Oluwafunke O. Nutrition education and counselling provided during pregnancy: effects on maternal, neonatal and child health outcomes. In: Database of abstracts of reviews of effects (DARE), Produced by the Centre for Reviews and Dissemination; 2013.

19. Rahman M, Abe SK, Kanda M, Narita S, Ota E, et al. Maternal anemia and risk of adverse birth and health outcomes: systematic review and meta-analysis. Hyderabad: Twenty-second Cochrane-Colloquium; 2014.

20. Scholl TO, Hediger M, Schall Jl, Khoo CS, Fischer RL. Dietary and serum folate: their influence on the outcome of pregnancy. Am J Clin Nutr. 2000; 63:520-5.

21. Fekete K, Berti C, Trovato M, Lohner S, Dullemeijer C, Souverein OW, Cetin I, Decsi T. Effect of folate intake on health outcomes in pregnancy: a systematic review and meta-analysis on birth weight, placental weight and length of gestation. Nutr J. 2012;11:75.

22. Palma S, Perez-Iglesias R, Prieto D, Pardo R, Llorca J, Delgado-Rodriguez M. Iron but not folic acid supplementation reduces the risk of low birth weight in pregnant women without anemia: a case-control study. J Epidemiol Community Health. 2008;62:120-4.

23. Khanal V, Zhao Y, Sauer K. Role of antenatal care and iron supplementation during pregnancy in preventing low birth weight in Nepal: comparison of national surveys 2006 and 2011. Arch Public Health. 2014:72(4).

24. Cogswell ME, Parvanta I, Ickes L, Yip R, Brittenham GM. Iron supplementation during pregnancy, anemia, and birth weight: a randomized controlled trial. Am J Clin Nutr. 2003;78:773-81.

25. Centeral Statitical Agency of Ethiopia: Ethipoia Demographic and Health Survey (EDHS) report on key findings,. 2016.

26. Beydoun H, Itani M, Tamim H, Alia Aaraj A, Khogali M, Yunis K. Impact of maternal age on preterm delivery and low birth weight: a hospital-based collaborative study of Nulli parous Lebanese women in greater Beirut. J Perinatol. 2004:24:228-35.

27. Khatun S, Rahman M. Quality of antenatal care and its dose-response relationship with birth weight in a maternal and child health training institute in Bangladesh. J Biosoc Sci. 2008;40:321-37. 